\title{
UNIQUENESS OF REPRESENTING MEASURES
}

\author{
FRANK FORELLI
}

\begin{abstract}
We improve a little an old theorem of Gleason and Whitney.
\end{abstract}
Let $\Delta$ be the maximal ideal space of the ring $H^{\infty}(\mathbf{D})$ of bounded holomorphic functions in the open unit disc $\mathbf{D}$. In this brief note we point out that if $\mu$ and $\tau$ are positive measures in $\Delta$ that represent the same point in $\mathbf{D}$, and if neither has any mass in $\mathbf{D}$, then $\mu=\tau$. A long time ago Gleason and Whitney proved that $\mu=\tau$ if neither has any mass off the Shilov boundary of $H^{\infty}(\mathbf{D})$. The point here is that $\mathbf{D}$ is smaller than the complement of the Shilov boundary.

LEMma. Let $\xi \in \mathbf{D}$ and let $\mu$ be a positive Borel measure in $\Delta$ with

(i) $\mu(\{|\hat{z}|<1\})=0$;

(ii) $f(\xi)=\int \hat{f} d \mu$ for every $f$ in $H^{\infty}(\mathbf{D}) . \quad(\hat{f}$ is the Gelfand transform of $f$.) Then

(iii) $\mu(\{|\hat{f}|<1\})=0$ if $f$ is inner.

ProOF. Let $f \in H^{\infty}(\mathbf{D})$; then

$$
f(z)=\sum_{j=0}^{\infty} \alpha_{j}\left(\frac{\xi-z}{1-\bar{\xi} z}\right)^{j}, \quad|z|<1 .
$$

Put $\varphi=(\xi-z) /(1-\bar{\xi} z)$. Then by $(1)$,

$$
f=\sum_{j=0}^{k} \alpha_{j} \varphi^{j}+\varphi^{k+1} f_{k+1},
$$

where $f_{k+1} \in H^{\infty}(\mathbf{D})$. This gives

$$
\int|\hat{f}|^{2} d \mu=\sum_{j=0}^{k}\left|\alpha_{j}\right|^{2}+\int\left|\hat{f}_{k+1}\right|^{2} d \mu .
$$

Here we use (i) and (ii) plus $\varphi(\xi)=0$. By (2),

$$
\sum_{j=0}^{\infty}\left|\alpha_{j}\right|^{2} \leq \int|\hat{f}|^{2} d \mu
$$

If $f$ is inner, the left side of this inequality is 1 . On the other hand, $|\hat{f}| \leq 1$ (if $f$ is inner). This proves (iii).

Received by the editors June 15, 1984.

1980 Mathematics Subject Classification. Primary $30 \mathrm{H} 05$. 
COROLlaRY. Let $\mu$ and $\tau$ be positive regular Borel measures in $\Delta$ that represent the same point in $\mathbf{D}$. If neither has any mass in $\mathbf{D}$, i.e. if both $\mu(\{|\hat{z}|<1\})$ and $\tau(\{|\hat{z}|<1\})$ vanish, then $\mu=\tau$.

ProOF. Put $\Omega=\bigcup_{f \text { inner }}\{|\hat{f}|<1\}$. If $F$ is a compact set in $\Omega$, then, by the Lemma, $\mu(F)=0$; hence, by regularity, $\mu(\Omega)=0$. Likewise $\tau$ has no mass in $\Omega$. But by Newman's theorem $[\mathbf{1}, \mathbf{3}$, or $\mathbf{4}]$

$$
\Omega^{\prime}=\text { the Shilov boundary of } H^{\infty}(\mathbf{D}) \text {; }
$$

hence, by the theorem of Gleason and Whitney $[\mathbf{1}, \mathbf{2}$, or $\mathbf{3}], \mu=\tau$.

By the Douglas-Rudin theorem the inner functions in $\mathbf{D}$ separate points in the Shilov boundary of $H^{\infty}(\mathbf{D})$. Using this and the fact that equality holds in (3) if $f$ is inner, one may give an alternative proof of the Corollary.

\section{REFERENCES}

1. J. Garnett, Bounded analytic functions, Academic Press, New York, 1981.

2. A. Gleason and H. Whitney, The extension of linear functionals defined on $H^{\infty}$, Pacific J. Math. 12 (1962), 163-182.

3. K. Hoffman, Banach spaces of analytic functions, Prentice-Hall, Englewood Cliffs, N. J., 1962.

4. D. Newman, Some remarks on the maximal ideal space structure of $H^{\infty}$, Ann. of Math. (2) 70 (1959), 438-445.

Department of Mathematics, University of Wisconsin-Madison, Madison, WISCONSIN 53706 